\title{
La comunicación comunitaria como clave reguladora privilegiada para las políticas nacionales de comunicación
}

\section{Community Comunication as a Privileged Regulatory Key for National Communication Policies}

\section{A comunicação comunitária como chave reguladora privilegiada para as políticas nacionais de comunicação}

\section{Adilson Vaz Cabral Filho}

Universidad Federal Fluminense - Brasil

\section{Páginas Cómo citar}

68-78 Cabral, A. (2017). La comunicación comunitaria como clave

DOI reguladora privilegiada para las políticas nacionales de http://dx.doi.org/10.26620/uniminuto. comunicación. Mediaciones, (19), 68 - 78. 
Resumen

El artículo a continuación reflexiona sobre la comunicación comunitaria como clave reguladora privilegiada para las políticas nacionales de comunicación; en él se plantea el problema de la comunicación comunitaria, aún de incipiente inclusión en el ámbito académico, y plantea alternativas que conduzcan a la interconexión de las políticas comunicativas con las políticas públicas y el desarrollo social comunitario, que se propone para la regulación del espectro electromagnético con participación del Estado.

En ese orden de ideas, se trata de diferenciar las estrategias adecuadas, de las fallidas, en los intentos de regulación en las políticas públicas de comunicación. Para ello el estudio reconoce tres modalidades en la aplicación de la regulación:

pública, privada y comunitaria; en el caso latinoamericano se registran avances en la legislación pero, también, existen contratiempos en las propuestas de Argentina y Brasil, referidas

a manera de ejemplo. En síntesis, el contexto actual necesita que los tres sectores coincidan en las necesidades de la regulación hacia un sistema de comunicación unificado, que facilite la integralidad de las políticas públicas sobre la comunicación.

Palabras clave Comunicación, políticas públicas, público, privado, 


\section{comunitario. Resumo}

\begin{abstract}
This paper presents a reflection on community comunication as a privileged regulatory key for national communication policies, whose inclusion in the academic field is still emerging. It suggests options leading to the interconnection of communicative policies with public policies and community social development, proposed for regulating the electromagnetic spectrum with the State's involvement. Thus, we try to distinguish appropriate strategies from unsuccessful ones, in the attempts to regulate public policies of communication. In order to do so, we identify three ways to apply regulation: public, private and community. In the Latin American case, there has been some progress in legislation, but there have been setbacks too in Argentina's and Brazil's proposal, just to mention some. Summarizing, current circumstances demand that the three sectors coincide in the need for regulation towards a unified communication system, which facilitates the integrality of public policies on communication.
\end{abstract}

\section{Key Words}

Communication, public policies, private, community.
O artigo a seguir reflete sobre a comunicação comunitária como chave reguladora privilegiada para as políticas nacionais de comunicação; nele se propõe o problema da comunicação comunitária, ainda incipiente, inclusão no âmbito acadêmico, e propõe alternativas que conduzam à interconexão das políticas comunicativas com as políticas públicas e o desenvolvimento social comunitário, que se propõe para a regulação do espectro eletromagnético com participação do Estado. Nessa ordem de ideias, trata-se de diferenciar as estratégias adequadas, das frustradas, nas tentativas de regulação nas políticas públicas de comunicação. Para isso o estudo reconhece três modalidades no aplicativo da regulação: pública, privada e comunitária; no caso latinoamericano registram-se avanços na legislação mas, também, existem contra tempos nas propostas da Argentina e Brasil, referidas a maneira de exemplo. Em síntese, o contexto atual precisa que os três setores coincidam nas necessidades da regulação para um sistema de comunicação unificado, que facilite a integralidade das políticas públicas sobre a comunicação.

\section{Palavras-chave}

Comunicação, políticas públicas, público, privado, comunitário. 


\section{Introducción}

La escasa visibilidad de la comunicación comunitaria, desde las políticas públicas, evita que se conozcan sus iniciativas. Este problema se manifiesta en la falta de incentivos gubernamentales para el sector y contribuye a distorsionar las profesiones que integran el campo de la comunicación, el cual podría incluir compañías que se distingan tanto en el sector privado comercial como en el sector público estatal. Tal reconfiguración representaría el mayor acceso al sector para estudiantes de pregrado en cursos de comunicación social y para personas en múltiples ocupaciones, con la capacidad de contribuir con su pluralidad y diversidad; mientras, se promueve una comunicación más democrática que se ratifique y asuma como derecho humano.

La omisión regulatoria es un problema que se origina en la limitada comprensión de la comunicación comunitaria en el ámbito académico, donde la comunicación se separa con frecuencia de sus políticas; así como, de los grupos sociales y de las organizaciones. Esto debido a una atención enfocada en las iniciativas comunitarias y no en las reglas que las soportan, cuando no se limita a un punto de vista instrumental de la actividad comunicativa. Cuando se trata de interconectar la comunicación comunitaria con las políticas comunicativas, debe asumirse el reto de contribuir a la formulación de alternativas regulatorias que ofrezcan un ambiente sostenible; mientras, se entiende el papel del Estado como regulador, gestor, inspector y evaluador, y se asumen facultades de apoyo y desarrollo, componentes de políticas públicas más amplias en un sector involucrado de forma directa con el desarrollo local y con políticas sociales asociadas con otros derechos humanos fundamentales.

Diversas iniciativas, propuestas en diferentes momentos y contextos, sirven de referencia a aquellos que las estudian o las orientan, para caracterizarlas. Se propone así la identificación de tales caracterizaciones en su contexto histórico, con el fin de contribuir al afianzamiento de caminos y patrones más adecuados, desde una perspectiva política que busque la regulación de dicho sector; por medio de, políticas nacionales de comunicación que legitimen, garanticen su sostenibilidad y hagan más efectiva su contribución al desarrollo social.

Por consiguiente, en este artículo se busca confirmar la suposición de que la comunicación comunitaria es una clave regulatoria privilegiada para la formulación de políticas nacionales de comunicación, que reconozcan la existencia de un sector comunicativo distinto al estatal y al comercial; en gran medida presentes en la legislación de diversos países en todos los continentes. 
Por otra parte, se emplea una metodología de investigación documental en relación con las regulaciones del sector de la comunicación comunitaria y de la comunicación en general; incluye la investigación bibliográfica, relacionada con los aspectos que se trabajan en el artículo, tales como la caracterización histórica de las iniciativas de comunicación hechas para y por segmentos sociales, enfocadas en el desarrollo humano en los lugares en que éstas operan y en el desarrollo social; por medio, de una movilización más amplia, tras su acción en diversos sectores sociales. Además de análisis relacionados con las iniciativas regulatorias en diversos países, con énfasis en la compresión de los sectores comunitarios sin ánimo de lucro, diferentes a los estatales y privados; y por último, lecturas que ofrezcan una contraposición crítica al concepto de tercer sector, que contribuyan a evidenciar los rasgos que lo distinguen de la comunicación comunitaria.

Los objetivos de este artículo son, en primer lugar, distinguir entre referencias adecuadas e intentos infructuosos de regulación, de manera que se establezca un diálogo con nociones teóricas relacionadas con otros adjetivos que caracterizan al campo de la comunicación y, por último, problematizar la existencia de un sector comunitario en el área de la comunicación, no como un tercer sector, ya que esto lo aproximaría a una perspectiva periférica impulsada por el mercado, a modo de iniciativa privada local, sino como refuerzo al amplio papel del Estado en la formulación de políticas públicas y democráticas para la comunicación, la cual se defienda y reafirme como derecho humano; en esencia, plural y diverso.

\section{El otro sector, en las políticas de comunicación pública}

El bajo costo de las tecnologías para producción de contenido y distribución en radio y televisión ha propiciado que las iniciativas de comunicación comunitaria se propaguen alrededor del mundo.
Esto pone de relieve la necesidad de una definición regulatoria por parte de un sector diferente al comercial privado o al estatal público; lo cual es, según el Informe McBride de 1968, una de las señales más relevantes de la transformación de las comunicaciones. La maduración de tales iniciativas, así como las radiodifusoras públicas estatales o privadas comerciales, han revelado otras necesidades relevantes; tales como, la reorganización del espectro electromagnético y de los fondos del Estado destinados a la comunicación.

Los conflictos regulatorios se presentan de diversas maneras, según los diferentes países y regiones que privilegian el sector público estatal o el privado comercial en sus políticas de comunicación. Además, existen claros términos y delimitaciones: un sector estrictamente diferente del binomio público privado, un servicio de utilidad pública, desde fondos públicos de apoyo hasta restricciones en la publicidad sin soporte del Estado, garantía de espectro de frecuencia, potencia y rango de transmisión limitado.

En 2007, la organización belga Kern European Affairs produjo el documento El estado de los medios comunitarios en la Unión Europea [The state of community media in the European Union], a solicitud de la Comisión de Cultura y Educación del Parlamento Europeo. El documento incluye un mapa del panorama europeo en cuanto a regulación del sector, con diferentes grados de reconocimiento para sus iniciativas en cada país (2007, pp. 11-18): normas por completo activas y relativamente bien establecidas o con la aprobación de cambios recientes en legislación y/o políticas; alta actividad, con reconocimiento legal a pesar de la ausencia de fondos públicos específicos; moderada a activa, que existe a pesar de la falta de un completo reconocimiento legislativo o de regulaciones relacionadas; y, acción limitada de los medios comunitarios o sin conclusiones significativas acerca de la actividad.

A su vez, el Programa de Legislaciones y Derecho a la Comunicación de AMARC-ALC (América Latina y el 
Caribe), comparó los marcos regulatorios de radiodifusión, de 29 países en los cinco continentes, con el propósito de identificar las prácticas legislativas y políticas públicas dirigidas a reconocer y promover la radio y la televisión comunitarias (Germano, 2010). Esta labor permitió la elaboración del documento Principios para un marco regulatorio democrático sobre radio y TV comunitaria, el cual guía las acciones de AMARc sobre la base de los temas propuestos a los gobiernos para la formulación de leyes democráticas para el sector, entre estos: diversidad de medios, contenido y perspectivas; reconocimiento y promoción; definición y características; objetivos y propósitos; acceso tecnológico; acceso universal; actividades competentes; procedimientos de concesión; requisitos y condiciones no discriminatorias; criterios de evaluación; financiamiento; recursos públicos; e, inclusión digital.

La investigación sintetiza la existencia de tres grandes sectores que configuran los sistemas de comunicación en los diferentes países en el estudio: el público (que en general se relaciona con el Estado), el privado y el comunitario. En algunos países, el número de licencias otorgadas a cada sector es el mismo, si bien ciertas diferencias, inherentes a cada contexto nacional, se mantienen. Estas incluyen los segmentos sociales que conforman las iniciativas, la extensión de sus poderes y la viabilidad del uso de publicidad que garantice su sostenibilidad.

Esta clase de estudios requiere una actualización permanente, con el fin de reconocer posibles fluctuaciones en los indicadores analizados para aspectos políticos, legales y socioculturales; mientras, se incluye la comunicación comunitaria en relación con el sistema de comunicación, de acuerdo con la lógica de la política económica y la estructura gubernamental de cada país.

Dentro de los modelos regulatorios más importantes, el europeo se caracteriza por ser predominantemente estatal. Sin embargo, en años recientes se han visto transformaciones asociadas con el avance del sector privado-comercial, el cual ha llegado a abarcar principalmente la entrada al sector de las telecomunicaciones, limitando incluso la comunicación pública-estatal. Es función, entonces, del sector comunitario reafirmar y luchar por políticas que garanticen su sostenibilidad.

A su vez, Estados Unidos tiene un sistema en su mayoría privado, que es referencia para otros países. Sin embargo, la movilización de una sociedad organizada en diferentes estados y la falta de regulación estricta en el sector, crea un espacio para una red público-estatal considerable; así como, una vía acceso a licencias de operación para iniciativas comunitarias urbanas.

En los años recientes, América Latina ha gozado de un tiempo oportuno con los gobiernos progresistas, tras haber avanzado en términos legislativos en algunos países. Aun así, se han presentado una serie de contratiempos, como en el caso de Argentina, en el actual proceso de desmantelar su Ley General de Servicios de Comunicación Audiovisual y de Brasil, donde los gobiernos progresistas desaprovecharon la oportunidad de promover los avances necesarios para la regulación del sector. Mientras una parte significativa de los países latinoamericanos concibe sus sistemas de comunicación en tres sectores, siendo uno de ellos comunitario pero la Constitución Federal brasileña, en su artículo 223, lo excluye de los tres sistemas que menciona: el privado, el estatal y el público ${ }^{1}$. Toda vez que el artículo no contiene una regulación específica, su significado e implicaciones se interpretan de diferentes maneras.

La Campaña por la Libertad de Expresión se lanzó en 2009, bajo la coordinación del Foro Nacional para la Democratización de la Comunicación, FDNc, junto con el Proyecto de Ley de Iniciativa Popular

1 Art. 5, - Como mínimo, 33\% de los canales o de la capacidad del espectro para radio y televisión se reservará para el sistema público, del cual, 50\% como mínimo, se destinará a servicios que entidades asociativas-comunitarias emitan. 
—PLIP-, sobre Comunicaciones; el cual, propone la conformación de un sistema de comunicación dividido en tres sectores; a saber, estatal, privado y público, en concordancia con la Constitución Federal actual, según la cual el sector público destina solo el 50\% de sus ayudas económicas a iniciativas comunitarias (Projeto, 2014); es decir, la mitad de lo que los países latinoamericanos lograron durante la primera década del siglo XX. El movimiento a favor de la aprobación de la PLIP derivó en una ruptura del derecho de la Conferencia Nacional de Comunicación como mecanismo co-administrativo que involucra, tanto al gobierno como a la sociedad; a pesar de, comprender la comunicación como derecho humano y reconocerla como instrumento de articulación social de otros sectores sociales tradicionales tales como la salud, la educación e incluso la cultura.

En contextos diferentes al brasileño, proyectos como los mencionados también presentan una dificultad conceptual de movimientos activistas, de comunicación y otros de naturaleza social, así como del sector académico mismo, para relacionar el sector con el proceso regulatorio del sistema de comunicación.

\section{El adjetivo que define la regulación}

Existen pocos adjetivos que se empleen con regularidad para describir el sector que se diferencia del público estatal y del privado comercial. Por décadas, quienes han intentado entenderlo mejor han considerado aspectos políticos organizacionales e identitarios empleando múltiples adjetivos propios de experiencias con diferentes propósitos, pero con una base común.

Las diferentes habilidades comunicativas desarrolladas por y para grupos populares son tan antiguas como las iniciativas mismas. Adjetivos tales como popular, participativo, horizontal, dialógico e, incluso, social denotan cierta clase de comunicación, que refuerza los atributos de articulación de los grupos involucrados o el propósito de los contenidos producidos. Estos refuerzan aún más las características que las cualidades de las iniciativas emprendidas y resaltan las contribuciones, por parte de grupos de apoyo y asesoría en relación con las personas, a quienes se adjudican los productos. Otras expresiones tan alternativas o independientes ratifican una dimensión política de los procesos y productos de comunicación, aun mientras enfatizan en una distinción que parte de su desprendimiento de los medios tradicionales; mientras, buscan maneras de poner en evidencia la administración, programación y producción, en especial de formatos de lenguaje y contenido relacionados con las personas. También, son empleadas en contextos mediáticos privados con diferentes estándares de calidad estética.

No sucede lo mismo con el uso de la expresión contrahegemónica, evidenciando el posicionamiento político frente a una forma específica de producción de comunicación, enfocándose en la política que sostiene y permite la continuidad de este modelo; destacando gerentes, programadores y productores en torno a este proceso. Boaventura de Sousa Santos (2014) lo entiende como «un trabajo organizado de movilización intelectual y política en contracorriente, con el propósito desacreditar los esquemas hegemónicos y proporcionar una comprensión alternativa y creíble de la vida social» (p. 33).

También, se debe notar que dichos adjetivos marcan una perspectiva de clase que caracteriza las prácticas de comunicación. Por tanto, la lucha contrahegemónica resulta en una búsqueda más amplia, mientras involucra no solo la afirmación de otra posible comunicación sino la comprensión de que diversas y múltiples iniciativas la exigen, comprenden, identifica y afirman. Tales iniciativas enfrentan un sistema consolidado, sostenido por un modelo de gestión y regulación que es más amplio que la comunicación misma, aun cuando esta lo legitima. 
También, se utilizan otras expresiones para referirse al propósito y a la contribución de las iniciativas sociales Comunicación para el desarrollo, C4D, y Comunicación para el desarrollo social, CfSC. Amparo Cadavid (2013) las expone de la siguiente manera:

La comunicación para el desarrollo hace referencia al campo de la comunicación en función de un modelo, como el desarrollo, y sitúa la comunicación como un instrumento al servicio del modelo. La comunicación para el cambio social señala la capacidad que proviene de la comunicación como campo de construcción social y cultural para transformar a toda la sociedad. (p. 29).

Los intentos de reacción más recientes a este modelo hegemónico han dado lugar a nuevos adjetivos durante las dos primeras décadas del siglo xxi. Los medios radicales (Downing, 2002) surgieron de grupos de población percibidos en procesos de enraizamiento social en sus lugares de acción, que conformaron sus propios espacios de acción reemplazando; en otras bases, las prácticas de comunicación populares en los años 70 y 80 .

Los medios tácticos, resultantes de la apropiación directa de tecnologías e infraestructuras de comunicación, potencian la producción y circulación de contenidos en tiempo real. Estas iniciativas promueven procesos organizados por colectivos de medios que incluyen el reciclaje de computadoras viejas para usos sociales; adoptan programas informáticos abiertos y gratuitos, basados en códigos abiertos, gratuitos y compartidos; abordan cuestiones políticas relevantes o provocan preguntas diversas a partir de intervenciones artísticas usando recursos multimedia variados y son entendidos como embriones de los movimientos de ocupación, en todo el planeta desde comienzos de la presente década.

La comunicación crítica también es un término que los activistas de medios reivindican en la actualidad, que comprende iniciativas y prácticas que afirman posiciones críticas en relación con los medios convencionales, pero que no es necesariamente un contrapunto al modelo contrahegemónico. Esto es suficiente para provocar reacción o reflexión, lo que lleva a una reorientación de las prácticas, tal como lo propone la Escuela Popular de Comunicación Crítica — EsPocc-, con sede en Maré, distrito de Río de Janeiro, la cual tiene la intención de difundir el trabajo de fotografía de sus estudiantes en suplementos de periódicos de gran circulación en el Estado.

Dentro de estos modelos contemporáneos, organizaciones semejantes a los circuitos de artistas frecuentes en la década de los setenta, surgen como una reorganización de grupos populares y sociales, mientras asesoran o no a sectores de población de bajos ingresos, promueven prácticas de comunicación que resultan en procesos interactivos e incluso colaborativos. Estos son:

[ ] nueva forma de acción iniciada por la resistencia joven, urbana y de clase media en el Brasil de los tiempos neoliberales [que] ganó cierta visibilidad a través de la discusión de la repolitización del arte y las nuevas formas de organización de las personas interesadas en manifestar su inquietud en relación a varios aspectos de la globalización neoliberal (Freire Filho y Cabral, 2008, p. 184).

En el campo más específico del activismo mediático, debe notarse que en el contexto latinoamericano, la radio siguió un camino diferente al de la televisión. Aunque similares, las radios se constituyeron en comunidad, en línea con la necesidad de regulación específica para tales iniciativas; a su vez, las iniciativas comunitarias en televisión surgieron a partir de prácticas populares de video movimiento, que tuvieron gran importancia en América Latina en la década de los ochenta, inicialmente funcionando en plazas y otros lugares públicos, y luego transmitiendo en experiencias locales. 
De toda esta amplia gama de posibilidades y referencias de calificación de activismo mediático, las iniciativas en torno a la radiodifusión comunitaria muestran trayectorias particularmente interesantes. Toda vez que están sujetas a regulación específica relevante al uso de espectro electromagnético, propiedad exclusiva del sector federal, se implementan durante el enfrentamiento con procesos regulatorios en países con mayor o menor restricción política.

Se entiende, por tanto, lo diferencial del término comunidad como una calificación regulatoria en relación con otros términos, con los que dialoga desde un punto de vista político e identitario. No se resta validez y contribución a estos adjetivos, los cuales definen y aportar un sentido sociocultural, relevante y pertinente a tales iniciativas.

Las críticas y reflexiones sobre los aspectos constitutivos de las iniciativas son necesarias dentro de una realidad compleja que involucra al sector, contribuyendo a generar referencias que fortalezcan los principios de compromiso con el desarrollo social; pero, entendidas entre las diversas posibilidades que conforman al ser comunitario: la falta de beneficio y proselitismo de cualquier tipo, mientras se busca fortalecer el carácter plural y diversificado de las iniciativas, el carácter local y en red de las diferentes localidades.

\section{Una comunicación comunitaria distinta del tercer sector}

La existencia de un sector comunitario en el área de comunicación no lo hace por fuerza un tercer sector, a pesar de la terminología que con frecuencia emplea la ideología neoliberal e incluso ciertos contextos sociales y académicos en políticas de comunicación, en especial en comunicación comunitaria. Quienes sostienen en sus críticas que las iniciativas desvinculadas del Estado o del mercado no pertenecen al tercer sector relacionan su punto de vista con el desmantelamiento del Estado, lo que intensifica la marginación de un sector que se asume como soporte de un estigma periférico.

El reconocimiento y la reafirmación del tercer sector como una expresión que se acepta en el campo político también conduce a la percepción de tres sectores independientes y no articulados; a la vez que, se descalifica la importancia del Estado, se respeta la penetración del mercado y se reconoce a la comunidad solo como un articulador de iniciativas plurales, diversificadas, participativas, horizontales, interactivas y dialógicas. Por tanto, se restringen a la comunidad muchos aspectos que deberían atribuirse al sistema de comunicación en su conjunto, constituyendo referencias legales para licencias de organismos de radiodifusión, participación en la gestión, programación y producción del sector, creación de redes, y acceso a formas de apoyo, entre otras áreas.

Tras definirse en la intersección entre el sector público y privado, el tercer sector se posiciona tanto para eliminar las limitaciones del Estado en términos de la satisfacción de las necesidades de la población como para crear un tipo de emprendimiento social precario, reduciendo así la importancia de la lucha de la clase trabajadora; aunque constituye, según las ong, una categoría profesional en sí misma. De acuerdo con la crítica de Montaño, "cuando se considera la sociedad civil como 'tercer sector', históricamente debería ser el 'primero'. Esta falta de rigor es poco importante solo para quienes no conciben la historia como un parámetro de la teoría» (2007, pp. 54-55).

Al diferenciarse de lo estatal y de lo privado, el tercer sector no asume especificidad pública, de servicio, interés y carácter, dada la inexistencia de criterios legales que determinen su gestión y sostenibilidad. La sociedad mantiene el Estado y puede aspirar a ser parte del mismo por medio de su participación en partidos políticos y en campañas dirigidas posiciones administrativas y estructurales. De la misma manera, mantiene el mercado al implementar 
empresas privadas. A diferencia de estas formas de organización, las iniciativas en torno a este otro sistema requerirían criterios que evidenciaran el interés y acceso de la comunidad, sin distinción, reconociendo sus particularidades en relación con la autonomía que afirman y sostienen.

La comprensión de lo público como independiente de lo estatal aún requiere que se supere el vínculo con propuestas neoliberales, como sugiere Bresser Pereira (1999), para quien uno de los principales desafíos de las ONG es «acercar sus funciones de promoción de la auto organización social a un carácter más empresarial». Dentro de este marco, lo que constituye lo público no estatal se convierte, en la práctica, en privado no comercial a escala local. En algunas ocasiones, no alcanza siquiera tal calificación. Dicha visión corrobora un desmantelamiento del aparato estatal, simultáneo a la privatización de actividades en áreas esenciales como la salud y la educación, lo que constituye un intento neoliberal de gestión a través de entidades del tercer sector. Por otro lado, también es necesario avanzar en la formulación de principios para su regulación en los contextos más diferentes, que incluyan los límites de lo que se comprende como beneficio dentro de estas iniciativas.

Más allá de las leyes, la vida cotidiana que sustenta y lleva a cabo estas iniciativas es aún más limitada, dada la necesidad de garantizar mecanismos que permitan e impulsen este sector sin fines de lucro. Tal como sostiene Morales (1998):

Si se entiende que la opción de política correcta para la descentralización administrativa es el tercer sector, esta asociación/colaboración debe expresarse en algo más que en relaciones contractuales. En este caso, el tercer sector debe recibir un tratamiento prioritario por parte del gobierno, como institución responsable del avance social [...]. Esto significa tratamiento institucional, financiamiento y regulación del sector en sí mismo, además del financiamiento y regulación de sus actividades. (p. 137).

Como sector independiente, el Estado garantiza la viabilidad de fondos a través de la implementación de políticas públicas, en vínculo directo con la voluntad política de los gobiernos actuales. A pesar de la comprensión de este conjunto de iniciativas como un tercer sector, tal como lo consideran organizaciones tales como AMARC y el Parlamento Europeo, es necesario reconocer las implicaciones de esta fragilidad constante, la cual asume grados de intensidad mayores o menores según las leyes gubernamentales y las iniciativas que garantizan su sostenibilidad.

\section{Conclusiones}

El complejo escenario actual permite cuestionar todo un sistema de comunicación que puede establecerse de otra manera, mientras se renegocian las relaciones entre lo público (institucional/estatal), privado (empresarial/comercial) y comunitario (sin fines de lucro/social). Cada vez es más claro que el sector privado no puede ser independiente del marco regulatorio, ni el sector público puede someterse al gobierno actual. De ahí la necesidad de que se entiendan como sectores interdependientes dentro del mismo sistema de comunicación, con reglas claras sobre una base común, en lugar de sistemas independientes como en Brasil.

Así como otros sectores, el comunitario exige visibilidad, reconocimiento, legitimidad y formas de sostenibilidad que tienen en el Estado un actor importante, al contar con las facultades para formular, implementar, vigilar y evaluar políticas públicas, así como supervisar e incluso, habilitar y fomentar iniciativas de comunicación comunitaria. Una perspectiva crítica que articule el campo académico y el social, en torno a la afirmación de la importancia del sector comunitario en la radiodifusión, debe buscar formular políticas que asimilen los cimientos de este otro sector al asignar 
definiciones claras para la distribución de los fondos gubernamentales y la división del espectro electromagnético, alrededor de un sistema de comunicación unificado que comprenda tres sectores interdependientes.

\section{Referencias}

Bresser-Pereira, Luiz Carlos e Grau, Nuria Cunill. (2012). Entre o Estado e o Mercado: o público não-estatal. In BresserPereira, L.C. e Nuria Cunill Grau (Orgs.). O Público Não-Estatal na Reforma do Estado. Rio de Janeiro: Editora FGV, 1999. Disponible en http://bresserpereira.org. br/papers/1998/84PublicoNaoEstataRef Est.p.pg.pdf.

Cadavid, A. (2013). La comunicación para el cambio social: ¿nos acerca o nos aleja? En Martínez Hermida, M.; Brandariz, J. A.; Lage, X.; Vázquez, M. (Coords.). Comunicación y desarrollo. Buenos Aires: La Crujía.

Dornelles, Beatriz. (2007). Divergências conceituais em torno da comunicação popular e comunitária na América Latina. Revista da Associação Nacional dos Programas de Pós-Graduação em Comunicação - COMPÓs. Disponible en http://www.compos.org.br/seer/index.php/ecompos/article/viewFile/ 176/177.

Downing, John D. H (2002). Mídia Radical: rebeldia nas Comunicações e Movimentos Sociais. São Paulo: Senac.

Festa, Regina E Lins Da Silva, Carlos Eduardo (orgs.) (1986). Comunicação Popular e Alternativa no Brasil. São Paulo, Paulinas.
Freire Filho, João e Cabral, Ana Júlia C. De B (2008). Contra-hegemonia e resistência juvenil: movimentos mundiais de contestação da ordem neoliberal. En Coutinho, Eduardo Granja (Org.). Comunicação e contrahegemonia. Rio de Janeiro: Editora UFRJ.

Germano Gómez, G. (2010). Principios para un marco regulatorio democrático sobre rádio y televisión comunitaria. In Gumucio-Dagron, Alfonso y Herrera-Miller, Karina. Política y legislación para la radio local en América Latina. La Paz, Plural.

Kern European Affairs (KEA) (2007). The state of community media in the European Union. Bruxelas, Parlamento Europeu.

Malerba, João Paulo. Rádios comunitárias: panorama da situação legal na América Sul. Publicado en mayo. 2012. Disponible en http://www. observatoriodaimprensa. com.br/news/view/_ed712_panorama_da_ situacao_legal_na_america_sul.

Montaño, Carlos (2007). Terceiro Setor e Questão Social: crítica ao padrão emergente de intervenção social. 4. Ed. São Paulo: Cortez.

Morales, Carlos Antônio. Nem privado nem estatal: em busca de uma nova estratégia para a provisão de serviços públicos. En Revista do Serviço Público. Año 49, Número 4 Oct-Dic 1998. Disponible en http://www. bresserpereira.org.br/ Documents/MARE/ Terceiros-Papers/98-Morales49(4).pdf

Santos, Boaventura de Souza (2014). Se Deus fosse um ativista dos direitos humanos. São Paulo, Cortez.

\section{Adilson Vaz Cabral Filho}

acabral@comunicacao.pro.br

Profesor asociado de la Universidad Federal Fluminense. 